\title{
GPR data interpretation in the landslides and subgrade slope surveys
}

\author{
Viktor Pupatenko ${ }^{1, *}$, Yuri Sukhobok ${ }^{1}$, Gennady Stoyanovich ${ }^{1}$, Andrey Stetsyuk ${ }^{1}$, and Leonid \\ Verkhovtsev $^{1}$ \\ ${ }^{1}$ Far Eastern State Transport University, 47 Seryshev St, Khabarovsk, 680021, Russia
}

\begin{abstract}
The paper describes results of GPR survey of multi-layered slopes of transport structures. A brief review of this problem by domestic and foreign researchers is presented. The significance of this work is determined by complexity of GPR data interpretation when the GPR survey is conducted in a common offset or common midpoint mode in case of dipping layers. The typical patterns are obtained, the groups of the same reflection events are picked and possible reasons of them are stated by numerical modeling using GprMax software. The technique of ground velocities finding in case of GPR survey of dipping layers is proposed. Keywords: GPR, common offset mode, multi offset mode, rock slopes, slope stability
\end{abstract}

\section{Introduction}

The ground penetrating radar (GPR) is one of the non-destructive geophysical methods. It is widely used in practice due to its high performance and cost effectiveness [1]. The most common GPR applications are the layer thickness measuring as well as the detection of cables, pipelines and other local subsurface objects [1,2,3]. GPR is widely used at all stages of new construction from road design to quality control.

There are some specific features of landslide slopes and subgrade slopes. Data processing and interpretation and even survey process often become very difficult due to complicated slope geometry, large number of multiple reflections, impossibility of multi-offset acquisition tests performance.

There are very few papers dedicated to the application of the GPR method to the unstable rock slopes surveying since the earliest one was written in 1995 [4]. Kulizhnikov and Eremin [5] proposed the landslide deformation forecasting method based on the interpretation of the returned GPR signal amplitude-frequency characteristics. In order to identify the potential dangerous landslide areas, the high-speed technique for GPR geotechnical inspection of roadways was developed by Benedetto et al. [6, 7]. Lissak et al. [8] demonstrated that GPR can be used to monitor the displacement of slow-moving landslides GPR data can be combined with other surveying techniques to estimate the vertical movement of rotational slides. Papers [9-11] present results of landslide slopes surveying in Germany, Switzerland and France with joint use of various geophysical methods, including GPR. Furthermore,

*Corresponding author: khv_pvv@mail.ru 
paper [12] describes the potential of the GPR method for assessing the rock-fall hazard in case of landslides monitoring in Austria.

Several sites with slope deformations on the Russian Far East railways and roadways were inspected by the Far Eastern State Transport University staff (Fig. 1, a). The main aim of the GPR survey was to detect layer boundaries and possible displacement surfaces.

(a)

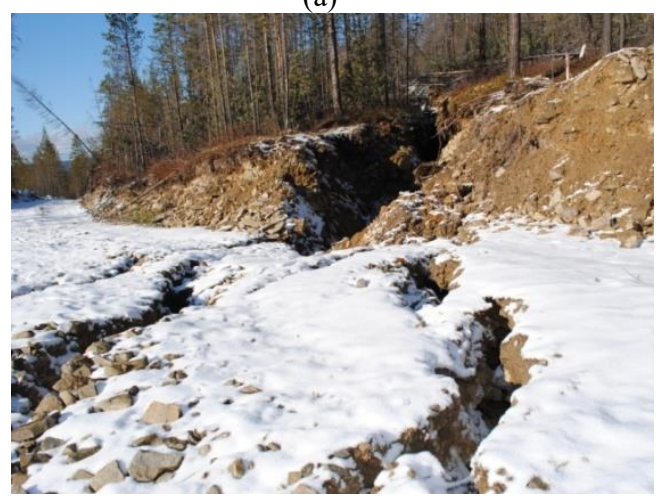

(b)

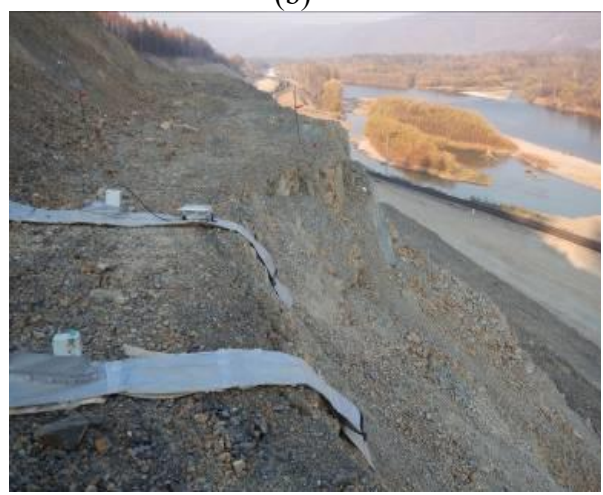

Fig.1. The top of the slope and the longitudinal cracks (a); GPR acquisitions on the steep slope (b).

The slopes are often covered with trees and their inclination reaches $1: 1$, therefore it was very difficult to carry out GPR tests along the surface. One possible solution of that problem is shown in Fig. 1, b. The 3 meters long low frequency antennas were lifted parallel to each other along the slope. The traces were recorded automatically with $1 \mathrm{~s}$ interval. In this case, one can define only the approximate position of each trace based on the total length of GPR profile. On all other profiles we manually checked the step of GPR unit using measuring tape.

\section{Materials and methods}

We used the LOZA-N GPR system, equipped with a $100 \mathrm{MHz}$ (1.5 m long) and $50 \mathrm{MHz}(3$ $\mathrm{m}$ long) antennas. The time-base was $512 \mathrm{~ns}$ and the sampling frequency was $1 \mathrm{GHz}$. The vertical resolution equals to a quarter of the signal wavelength. The last one is expressed as follows $[1,2]$ :

$$
\lambda=\frac{\mathrm{c}}{3 \mathrm{f}_{\mathrm{c}} \sqrt{\varepsilon_{\mathrm{r}}}}
$$

where $\mathrm{c}$ is the free space electromagnetic propagation velocity $(0.3 \mathrm{~m} / \mathrm{ns}), \mathrm{f}_{\mathrm{c}}$ is the central frequency of the signal $(\mathrm{GHz})$ and $\varepsilon_{\mathrm{r}}$ is the real part of the dielectric permeability of the soil. The horizontal resolution depends on the first Fresnel zone radius [15]:

$$
\mathrm{R}_{1}=\frac{\sqrt{\mathrm{R \lambda}}}{2}
$$

where $\mathrm{R}$ is the penetrating depth (m).

According to (1) the vertical resolution is about $0.4 \mathrm{~m}$.

The slope deformation leads to the formation of a vertical cracks with a breadth of up to $4 \mathrm{~m}$. In this case reflections on the radargram are recorded in the form of straight inclined lines and also as a complex combination of hyperbolas in the lower part of the profile. The interpretation of such radargrams is complicated by the presence of re-reflections from inhomogeneities, air reflections from slopes, trees and other objects.

We used the KROT, MatGPR [15] and GeoReader [16] software to process field radargrams. We used gprMax software [17] to calculate synthetic radargrams. 
We assume in our models that the crack is filled with air, thus the signal in the radargrams is high-contrast. We assume that the crack extends to the competent layer boundary, but in our models we also examine intermediate variants with gradual development of the crack in the weak layers. The main aim of analysis and interpretation of syntetic radargrams was to learn how to restore the radio wave velocity of the soils that the crack crosses.

\section{Numerical modeling results}

We calculated synthetic radargrams for three models of a layered medium with a vertical crack (fig. 2). The layers' thickness was set to 4,5 and $5 \mathrm{~m}$, respectively. The radio wave velocity was set to $0,1 \mathrm{~m} / \mathrm{ns}$ in the first and third layer and $0.15 \mathrm{~m} / \mathrm{ns}$ in the second layer.

(a)

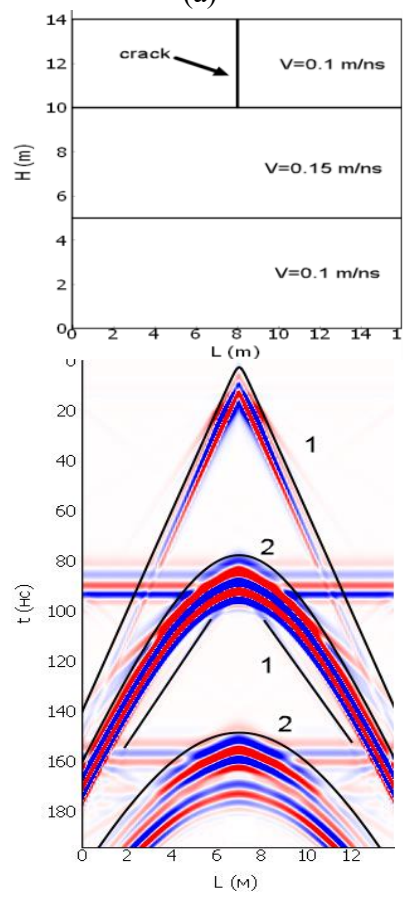

(b)
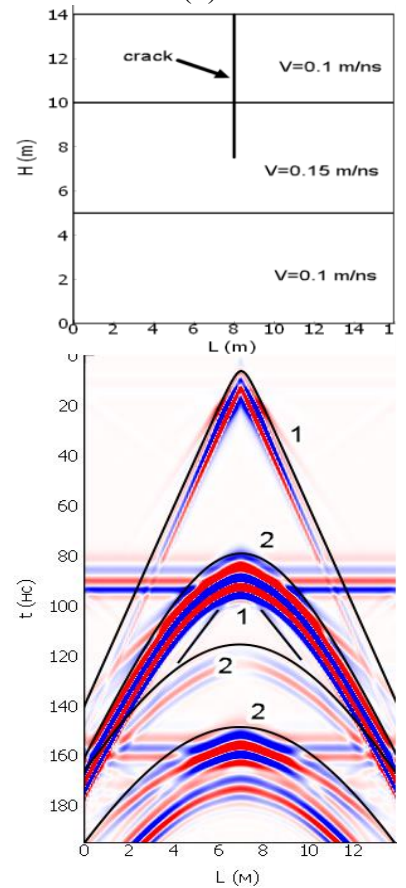

(c)

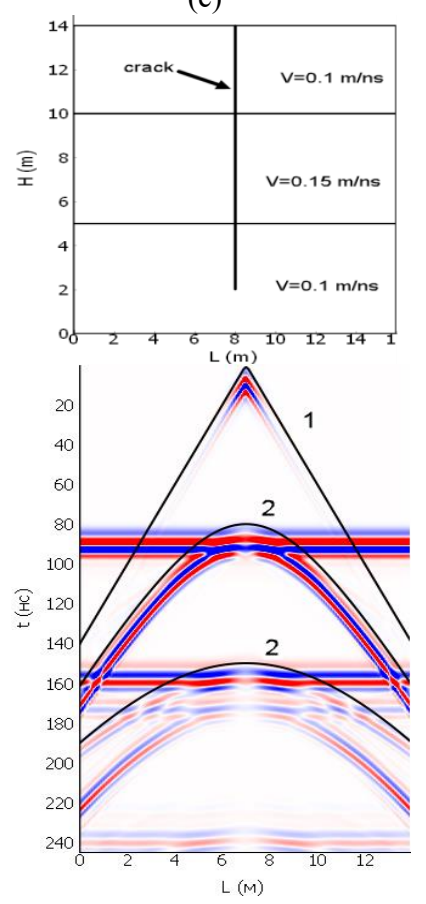

Fig. 2. Models of layered medium with a vertical crack and corresponding synthetic radargrams. 1 wave reflected from vertical crack boundary; 2 - hyperbolic reflections.

We found specific reflection types on all synthetic radargram. The first reflection type is associated with the reflected wave from the vertical boundary of the crack. The line inclination angle corresponds to the propagation velocity of radio waves in the layer. These reflections are less noticeable in the last two models due to a decrease in signal amplitude at the upper layers' boundary by a factor of 30 . When a crack intersects each layer boundary, hyperbolic reflections is formed. We can use these reflections to calculate depth-weighted average propagation velocity of radio waves. For the example in Fig. 2, they are, respectively, $0.1 \mathrm{~m} / \mathrm{hs}$ and $0.125 \mathrm{~m} / \mathrm{ns}$. These data allow us to calculate velocities in the lower layers and move from the time profile to the deep one.

To clarify the possibility of creating time-distance curves on slopes, calculations were performed using WARR technoque (fig. 3, a, b, c). In the first model, the profile is located on a horizontal platform in the upper part of the slope. The results are shown in fig. $3, \mathrm{~b}$. Calculation of the speed model is carried out according to the traditional method [18]. The time-distance curve equation is written as follows: 


$$
t(x)=\sqrt{t_{0}^{2}+\left(\frac{x}{V_{R M S}}\right)^{2}},
$$

where $t_{0}$ is the position of the time-distance curve vertex (ns), $x$ is the distance between antennas $(\mathrm{m})$ and $V_{R M S}$ is the average wave propagation velocity in a medium $(\mathrm{m} / \mathrm{ns})$.

(a)

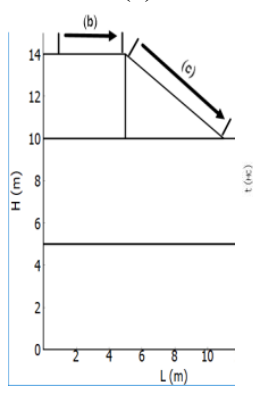

(b)

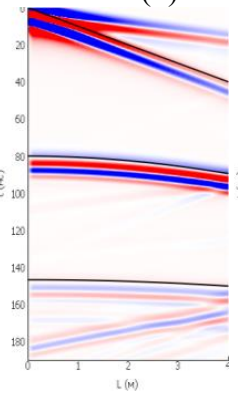

(c)

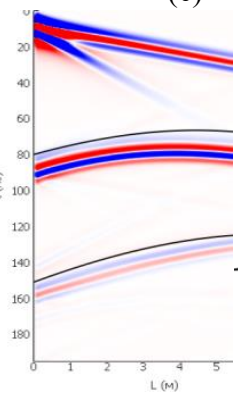

(d)

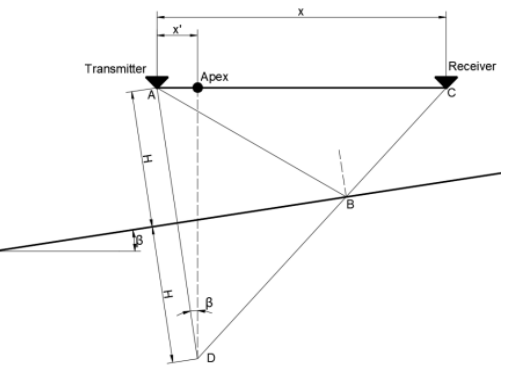

Fig. 3. Modelling results (WARR technique): (a) the calculation scheme; (b) simulation results at the main site and (c) on the slope (the black lines indicate hyperbolic time-distance curves of reflected waves); (d) the design scheme of wave propagation in a two-layer medium with an inclined reflecting boundary.

When the time-distance curve on the slope surface is calculated, the vertex of the hyperbolic time-distance curve of the wave, reflected from the inclined surface, is displaced by an amount of $x^{\prime}[14]$ :

$$
x^{\prime}=2 H \sin \beta
$$

where $\beta$ is the inclination angle of the layer's boundary and $H$ is the depth of the layer determined perpendicularly to the surface of the layer and at zero distance between the receiving and transmitting antennas.

The equation of the time-distance curve in this case is written as follows:

$$
t(x)=\frac{\sqrt{x^{2}-2 \cdot x \cdot x^{\prime}+t_{0}^{2} \cdot V_{R M S}^{2}}}{V_{R M S}} .
$$

Both approaches to the travel time curves processing are implemented in the GeoReader software [16].

\section{Practical applications of the results}

The practical result of the proposed methods for interpreting GPR data is shown in Fig. 5. Deformations of the slope led to the development of a system of cracks with a depth of up to $10 \mathrm{~m}$. The crack opening varies from 0.2 to $3.5 \mathrm{~m}$. The rupture on the radargram corresponds to the position of the largest crack (Fig. 1, a). 
a)

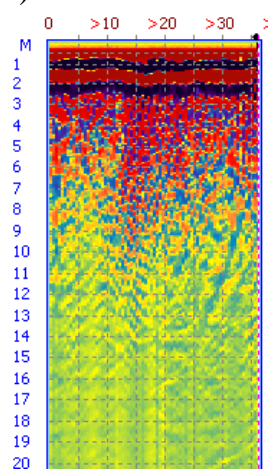

b)

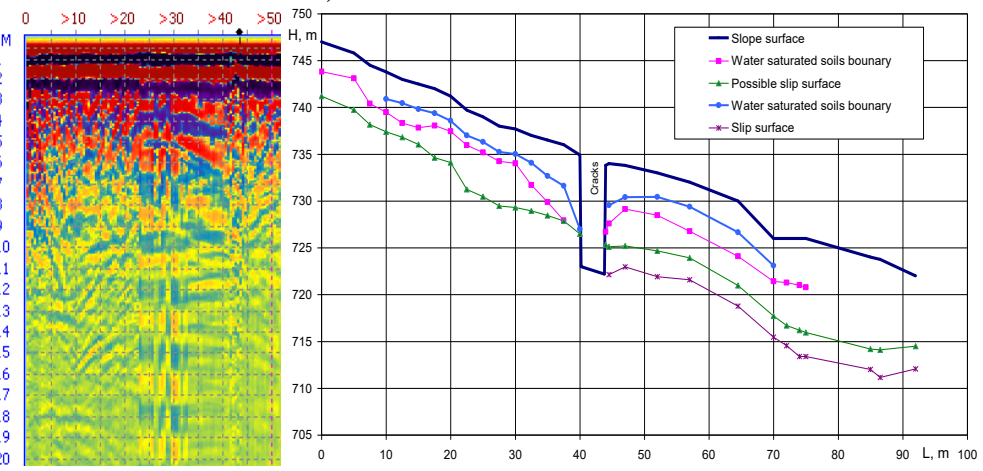

Fig. 4. Radargrams example (a) and geological cross-section with topographic reference (b).

We found several boundaries on the geological cross-section. There are two boundaries below the crack, along which slope displacement is possible. The velocity characteristics of soils were first determined using reflections from cracks and then refined by the travel time curves processing. Then we formed the topographic reference for the cross-section and switched it from time to depth. The obtained results allow to determine the volume of the landslide massif, and also provide the initial information for the slope stability calculation.

\section{Conclusions}

The results of the GPR survey allowed us to clarify the design model of the landslide slope and to reveal the most dangerous displacement surface. Stability calculations are performed for all possible combinations of displacement surfaces. The calculated characteristics of the soils were determined assuming the ultimate slope condition.

The results are applicable for determining the stability factor of landslide slopes, with the possibility of taking into account the additional vibrodynamic impact transferred to the ground objects from modern locomotives and mobile units with high axial loads.

\section{References}

1. H.M. Jol, Ground Penetrating Radar Theory and Applications (2009)

2. D.J. Daniels, Ground Penetrating Radar (2004)

3. A. Benedetto, L. Pajewski, Civil engineering applications of Ground Penetrating Radar (2015)

4. T. Toshioka, T. Tsuchida, K. Sasahara, Journal of Applied Geophysics 33, 119-124 (1995)

5. A.M. Kulizhnikov, R.A. Eremin, Surveying of landslide massifs of subgrade slopes by the ground penetrating radar method, 8th EAGE Int. Scientific and Practical Conf. and Exhibition on Engineering and Mining Geophysics, 54-64 (2012)

6. A. Benedetto, F. Tosti, International Workshop on Advanced Ground Penetrating Radar, 1-6 (2011)

7. A. Benedetto, F. Benedetto, F. Tosti, Nondestructive Testing and Evaluation 27-3, 253262 (2012)

8. C. Lissak, O. Maquaire, J.-P. Malet, et.al. Nat. Hazards Earth Syst. Sci. 15, 1399-1406 (2015) 
9. S. Carpentier, M. Konz, R. Fischer, G. Anagnostopoulos, K. Meusburger, K. Schoeck, Journal of Applied Geophysics 83, 46-56 (2012)

10. D. Jongmans, S. Garambois, Bull. Soc. géol. 178-2, 101-112 (2007)

11. O. Sass, R. Bell, T. Glade, Geomorphology 93, 89-103 (2008)

12. K.-H. Roch, W. Chwatal, E. Brück, Landslides 3, 87-94 (2006)

13. V.V. Pupatenko, Y.A. Sukhobok, G.M. Stoyanovich, Procedia Engineering, Transportation Geotechnics and Geoecology 189, 643-649 (2017)

14. V.V. Pupatenko, Y.A. Sukhobok, G.P. Sokolov, K.V. Pupatenko, Slip Surface Identification when Deformations of cutting slopes, Proc. of the $8^{\text {th }}$ Int. Scientificallypractical Conf. Transport Infrastructure of the Siberian Region, 485-490 (2017)

15. A. Tzanis, FastTimes 15-1, 17-43 (2010)

16. Y.A. Sukhobok, V.V. Pupatenko, A software tool for ground penetrating radar data processing, Proceedings of the Third All-Russian Information Technology and Highperformance Computing Conference, 175-180 (2015)

17. C. Warren, A. Giannopoulos, I. Giannakis, Computer Physics Communications 209, 163-170 (2016)

18. Y.A. Sukhobok, V.V. Pupatenko, G.M. Stoyanovich, Y.V. Ponomarchuk, Procedia Engineering, 143, 1236-1243 (2016) 